\title{
Linguistic and Personological Features of Suicidality In Bulimia: A Study of Memoirs \\ Sara Cantu ${ }^{1}$ and Cass Dykeman Oregon State University
}

\begin{abstract}
Bulimia and suicide ideation and behavior co-occur at rates that warrant investigation. This study applied a corpus linguistic tool to examine memoirs of people self-identifying as having been diagnosed with bulimia. A log likelihood statistic was used to determine word usage rates in the memoir corpus compared to known norm rates. Besides $p$ values, effect sizes (i.e., Bayesian Information Criterion) were reported for all inferential analyses. The study found that memoirs of people identifying as having been diagnosed with bulimia included differences in various linguistic processes and psychological processes. In addition, the usage rates of words associated with the core dimensions of Interpersonal-psychological theory of suicide were described. Keywords: Bulimia, Suicide, LIWC, Corpus Linguistics, Interpersonal-Psychological Theory of Suicide, Joiner, IPT, Personological
\end{abstract}

\section{Introduction}

The Centers for Disease Control and Prevention (2018) reported that suicide is the tenth leading cause of death in the United States, the second leading cause of death in people between the ages of 10 and 34, and the fourth leading cause of death in people ages 35 to 54 . There are twice as many suicides per year than homicides in the United States. In Alison Wertheimer's (2014) book, A special scar: The experiences of people bereaved by suicide, she wrote, "In most cases, suicide is a solitary event and yet it is often far-reaching repercussions for many others. It is rather like throwing a stone into a pond; the ripples spread and spread" (p. 5 ). The number of people impacted by the effects of suicide are immeasurable. This impact emphasizes the need to shape treatment and intervention to better suit the needs of people who are vulnerable to suicide. This study uses language analysis tools to grow the understanding clinicians have of dynamics impacting people diagnosed with eating disorders and suicidality.

The Centers for Disease Control and Prevention (2018) reported that 45,000 died as a result of suicide in 2016. In addition to completed suicides, about 13 million Americans will attempt suicide in their lifetime (Coppersmith, Dredze, Harman, \& Hollingshead, 2015). Eating disorders have the highest mortality rate of all the mental health diagnoses (Arcelus, Mitchell, Wales, \& Nielsen, 2011). Suokas et al. (2014) reported that someone with an eating disorder diagnosis has a risk of death of $12.8 \%$, suicide being the primary cause of $45 \%$ of these deaths. There is an estimated 30 million people in the United States that meet criteria for an eating disorder during their lifetime (Hudson et al., 2007). The number of people diagnosed with an eating disorder impacted by suicide or a suicide attempt is significant and warrants a need to better understand suicidality and eating disorders. Joiner's (2009) interpersonal theory of suicide holds that three elements drive the move toward suicidal ideation and behavior: (1) perceived burdensomeness, (2) thwarted belongingness, and (3) acquired capacity for suicide. Joiner (2009) held that these elements are key to appropriate identification, prevention,

1. This preprint has not yet been peer-reviewed. All comments are welcomed and can be sent to the first author at cantus@oregonstate.edu 
and treatment of suicidality in people diagnosed with eating disorders, including bulimia nervosa. Despite the predictive power of these three, knowledge of how they impact language is non-existent. Fortunately, technology has allowed for the advancement of psychological study of language. Using programs such as LIWC, connections between language used and behavior personality cognitive and emotional styles can be better understood (Pennebaker, 2010). With this increased understanding, there is a potential for the development of more effective interventions.

A review of the literature on the relationship between the interpersonal theory of suicide and eating disorders reveals these dominant themes: (1) the influence of perceived burdensomeness, thwarted belongingness, and acquired capacity for suicide on suicide; (2) the physiological impacts of bulimia that lead to suicidal ideation; (3) bulimia and suicide rates; (4) memoirs as sources of rich text; and (5) the need for an appropriate tool with which to assess risk factors identified by the interpersonal theory of suicide. After addressing these five themes, the research questions that guided this study will be stated.

Through the interpersonal theory of suicide, Joiner (2009) explained that people are at greater risk for suicidal behavior when they feel an increased sense of perceived burdensomeness, thwarted belongingness, and acquired capacity for suicide. Perceived burdensomeness is the perception that a person is an inconvenient obligation to those around them. Thwarted belongingness can be described as a diminished feeling of social connectedness and social support and a sense of loneliness (Hill \& Pettit, 2014). Studies have found that someone with a mental health diagnosis that involves symptomology leading to dependency on others, social withdrawal, and social skill deficits is more likely to experience perceived burdensomeness and thwarted belongingness to a greater degree and has an increased risk of suicide (Silva, Ribeiro, \& Joiner, 2015). Perceived burdensomeness and thwarted belongingness cannot in isolation predict suicide. Joiner (2009) clarified that someone must have the ability to die by suicide as well as the desire to die by suicide in order to complete suicide. The ability to die by suicide - acquired capacity for suicide - is a dampened reaction towards dangerous situations or risky behavior gained through life experiences. Joiner (2009) explained acquired capacity for suicide can be gained through fear-inducing situations, risky behaviors, such as extreme sports, non-suicidal self-injury, and exposure to violence. Understanding perceived burdensomeness, thwarted belongingness, and acquired capacity for suicide is key to addressing suicidality effectively. By addressing these factors, clinicians decrease the likelihood of suicide in clients, including someone diagnosed with an eating disorder.

Eating disorders, including bulimia nervosa, involve alienating and hazardous behavior and symptoms, as well as a sense of isolation through the course of treatment. Bulimia behavior patterns involve overeating, under eating, induced vomiting, and in some cases using a form of laxative. Medical complications involved in bulimia can include: alopecia; xerosis; lanuginose; brittle hair and nails; broken blood vessels in ears, nose, and eyes; erosion of teeth; enlargement of salivary glands; acid reflux; enflamed vocal cords and larynx; polyps; ulcers and cancer of the esophagus; severe dehydration; edema; arrhythmia of the heart; congestive heart failure; increased risk of miscarriage; aspiration of food; loss of normal colon functioning including severe diarrhea and constipation; bone loss; diabetes; and high cholesterol (Mehler \& Rylander, 2015; Sagar, 2005). This symptomology and behavior have the potential to increase feelings of perceived burdensomeness, thwarted belongingness, and acquired capacity (Brausch \& Perkins, 2018). Specific understanding of variables detailed in Joiner's (2009) interpersonal theory of suicide (IPTS) and symptomology involved in a diagnosis of 
bulimia increases the ability of mental health clinicians to address this concern through the course of treatment.

Given the frequency of bulimia, it is important to understand the impact of suicide on this population. The rate of suicide and self-harming behaviors in someone diagnosed with an eating disorder is higher compared to health control groups as well as other psychiatric diagnoses (Arcelus et al., 2011). Additionally, completed suicide occurs more often in people diagnosed with anorexia compared to people diagnosed with bulimia, and suicide attempts occur more often in people diagnosed with bulimia compared to anorexia (Forcano et al., 2009). These data highlight that there remains missing information in the field to best understand suicide attempts and completed suicide in people diagnosed with bulimia. Additional detailed information is needed to inform best treatment practices.

The language we use gives insight into what we are thinking, how we are feeling, and the focus of attention. Positive political ads include more present and future tense verbs compared to negative political ads that include more past tense verbs (Gunsch et al., 2000) Personal pronouns provide information on the writer's focus in the examples of people in pain or people asked to described their experiences (Tausczik \& Pennebaker, 2010). Fernández-Cabana et al. (2013) found a higher percentage of first-person singular pronouns and lower percentage of first-person plural pronouns reflect a greater sense of social isolation and an increased sense of thwarted belongingness, and that the use of third person pronouns indicate degree of flexibility and positive coping. Memoirs are a source of complex, multifaceted information from which we can draw. There is opportunity to examine and learn from the language used in memoirs to gain greater understanding of the relationship between suicide and someone diagnosed with bulimia.

There is a need for an effective means of evaluating Joiner's (2009) IPTS risk factors in distinctive populations, such as people diagnosed with eating disorders. Text analysis tools have been used recently to identify and better understand themes in a variety of populations and in different contexts. Coppersmith, Ngo, Leary, and Wood (2016) found that there was a significant difference between language used by someone who has attempted suicide compared to someone of the same age and gender who has not attempted to suicide. Handelman and Lester (2007) identified a difference between the language used in the suicide notes of attempters versus completers. Linguistic analysis is useful in understanding characteristics of suicide. Text analysis tools, such as LIWC, have the potential to uncover more of the dynamics of suicide and its impact on people diagnosed with eating disorders.

Given the aforementioned gaps in the literature on eating disorders and suicidality, four questions were developed to guide this study. The first research question was: What are the usage rates for linguistic and psychological processes related to suicidality? The second research question is: Do linguistic processes word usage rates differ from known norms? The third research question is: Do psychological processes word usage rates differ from known norms? The fourth research question is exploratory in nature: What are the usage rates for words in the following areas: (a) thwarted belongingness, (b) perceived burdensomeness, and (c) acquired capacity?

\section{Method}

\section{Design}


This study utilized a synchronic corpus linguistic design (Weisser, 2016). The unit of analysis was single words. The variables included: first-person singular pronouns, thirdperson singular pronouns, first-person plural pronouns, third-person plural pronouns, positive emotion, negative emotion, anxiety, anger, sadness, risk, biological processes, perceived burdensomeness, thwarted belongingness, and acquired capacity. All variables were non-negative discrete. The corpus was composed of memoirs written by people who self-identify as having been diagnosed with bulimia. The unit of analysis was single words for all research questions. Each memoir was examined separately. The analysis results of each sub corpus were then averaged to determine results for the corpus.

For the second and third questions, an a priori power analysis for chi-square goodness of test was completed by employing G*Power 3.1.9.2 (Faul et al., 2009). The effect size for this type of analysis is Cohen's $w$ (Cohen, 1992). The specific effect size was the average Cohen's $w$ reported by a study on the relationship of personality and suicidality (Lewitzka, Denzin, Sauer, Bauer, \& Jabs, 2016). The input parameters were: (a) test family $=2$; (b) statistical test $=$ goodness of fit tests: contingency tables; (c) type of power analysis $=$ a priori: compute required sample size given $\alpha$, power, and effect size; (d) $w=0.21$; (e) power $(1-\beta$ err probability) $=0.90$; (f) $\alpha=.05$; and (g) degrees of freedom $(d f)=1$. The $\mathrm{G}^{*}$ Power output noted a sample size of 239 and an actual power of 0.90 .

\section{Corpus}

Register, Scope and Sources. The corpus for the present study was made up of published memoirs written by an author self-identified as having been diagnosed with bulimia. Memoirs excluded from the corpus include: (1) published in a language other than English, (2) self-help books, and (3) books written by more than one author. It should be noted that the diagnosis was based upon self-identification rather than a formal $D S M$ diagnosis. To construct the corpus, researchers intended to use memoirs that were included on a list of books recommended by the Eating Disorder Resource Catalogue published by Salucore LLC, a publishing company specializing in eating disorder publications and education since 1980. In order to achieve adequate statistical power, it was necessary to augment the corpus with memoirs meeting the criteria for the corpus not on the recommended reading list. Additional memoirs were found on a list published by bookriot.com of 50 must-read books about eating disorders. The final selection of books included Armstrong (2009), Gay (2017), Holme (2012), Marnell (2017), and Waters (2011).

Preprocessing. The software used in this study to convert .kfx files to plain text was Kindle Converter v3.19.918.386 (2019). After preprocessing, the corpus contained 234,987 words and 22,666 types. At the memoir level, the word counts ranged from 58,829 to 122,326 and 4,841 to 10,749 types.

\section{Measures}

Linguistic Inquiry And Word Count (LIWC- Pronounced "Luke"). LIWC2015 contains a dictionary of approximately 6,400 words. This dictionary is divided into 90 subdictionaries, which may also be referred to as variables. The linguistic scales chosen for this study have been associated with a person's level of social connectedness. The scales used were: first-person singular pronoun, third-person 
singular pronoun, first-person plural pronoun, and third-person plural pronoun. The psychological variables used for the study included: positive emotion, negative emotion, anxiety, anger, sadness, and risk. In addition, a variable using biological processes was employed. The LIWC psychometric manual reports adequate validity and reliability for this measure (Pennebaker, Boyd, Jordan, \& Blackburn, 2015).

Norm Comparison Group For Second And Third Research Questions. The LIWC psychometric manual reports a variety of norms for different registers. Two relevant register norms for the present study were expressive writing and novels. The expressive writing norms had a significantly lower word count compared to the memoir corpus. The novel norm was used to provide a more equitable comparison. Because the exploratory custom dictionaries developed for this study are not part of the preset LIWC subdictionaries, no norm comparison analyses could be completed with these custom dictionaries.

Exploratory Custom Dictionaries. Custom dictionaries were developed for the fourth research question. The goal of this question was exploring the relationship of LIWC subdictionaries to core IPT dimensions. The contents for the custom dictionaries were culled from academic writing on bulimia and suicide as well as the clinical experience of the authors.

Thwarted Belongingness Dictionary (TBD). The TBD contains words that convey feelings of separation from others. A feeling of connectedness to the world works as a protection, safeguarding them from the isolating feelings that can contribute to lethal suicide attempts. This dictionary can be found in Table 1.

Perceived Burdensomeness Dictionary (PBD). The PBD contains words that illustrate a mindset of diminished self-worth, a belief that a person's environment would be better off without them. Someone with a high degree of perceived burdensomeness believes that they have a negative impact on the people around them, and that those people would be better off if they were gone. This dictionary can be found in Table 1 .

Acquired Capacity For Suicide Dictionary (ACSD). The ACSD contains words that capture the developed ability to overcome one's natural protective response to danger and fear. The words chosen for the ACSD are specific to the experiences of people diagnosed with eating disorders. Some of the words included in the dictionary are applicable to other populations with an increased risk for suicide. Other words included have specific significance to the eating disorder population. This dictionary can be found in Table 1.

\section{Apparatus}

LIWC. The software used in this study to analyze the data was the Linguistic Inquiry and Word Count (LIWC; Pennebaker, Booth, et al., 2015). LIWC's most recent revision, LIWC2015, will be used for this study. LIWC2015 features the option to create custom dictionaries, and one specific to the eating disorder population was created for this study (Pennebaker, Booth, et al., 2015).

Kindle Converter. The software used in this study to convert .kfx files to plain text was Kindle Converter v3.19.918.386 (2019). Once files were converted to plain text files, the files were compatible for use with LIWC. 


\section{Data Analysis}

For the first and fourth question, the across memoirs averages for raw count and percentage of all words were reported for each variable. For the second and third research questions, the log likelihood statistic $(L L)$ was used to assess whether average word usage rates in the memoir corpus significantly differ from corresponding known norm rates. In addition to $p$ values, effect sizes for each variable will be provided (i.e., Bayesian Information Criterion, BIC). BIC strength descriptors were drawn from Fabozzi, Focardi, Rachev, and Arshanapalli (2014). All analyses were conducted using R with a family-wise error rate $=.05$ and hence, an adjusted error rate $=.0045$.

\section{Results}

Both absolute and relative average usage rates for all variables appear in Table 2. Multiple significant results were encountered for the second and third research questions. Detailed results for these research questions including effect sizes appear in Table 3. For the variables that were statistically significant, the obtained effect sizes were strong (i.e., negative emotion) or very strong (i.e., first-person singular word use, third-person singular word use, third-person plural word use, biological processes). The fourth research question was exploratory in nature. The usage rates for the custom dictionaries of thwarted belongingness, perceived burdensomeness, and acquired capacity appear in Table 4.

\section{Discussion}

This study aimed to examine word usage in memoirs written by people diagnosed with bulimia. Linguistic Inquiry Word Count (LIWC) was used to analyze linguistic and psychological processes of the corpus, as well as word usage related to the interpersonal theory of suicide (Joiner et al. 2009). Results of this study inform the way that clinicians work with clients that have been diagnosed with bulimia.

The results from the first question reveal higher use of first-person language compared to third person language. One explanation for these results is that someone diagnosed with bulimia lives in isolation. An alternative explanation for the results encountered is a greater focus on self and a sense of isolation rather than awareness or focus on the collective. Between the former and the latter explanations, the latter is the most likely because the writers involved in the corpus did not live in isolation, likely similar to other people diagnosed with bulimia, and in fact had supports around them. An increased focus on self implies an increased feeling of thwarted belongingness, putting them at greater risk of self-harm or suicide.

Findings from the second question, show greater use of first person singular, first person plural and third person plural in the corpus compared to the novel norm. One probable explanation for the obtained results is that someone without a diagnosis of bulimia has a greater sense of belonging and connectedness to people around them. A competing explanation is that someone with a diagnosis of bulimia has a greater sense of self-importance compared to someone without a bulimia diagnosis. Among these probable reasons, the initial explanation is most likely the correct one because bulimia is characterized by self-dissatisfaction with an emphasis on body (Miller \& Golden, 2010) not over confidence. An increased sense of social isolation, potentially related to feelings 
of disconnection as a result of behavior related to bulimia as well as treatment for bulimia, has been identified in previous research to be the greatest indicator of suicidality (van Orden et al., 2010). It is reasonable to expect that the writing of someone diagnosed with bulimia would reflect a greater sense of isolation and therefore greater risk for suicidality compared to the norm given the increased risk of suicide in someone diagnosed with bulimia (Arcelus et al., 2011).

The psychological domains of the corpus compared to the psychological domains of the norm reflect significantly more negative emotion words and biological words used in the corpus. A potential explanation for the results is people diagnosed with bulimia are significantly more focused on their body and experience anxiety and depression at a higher rate than average. An alternative explanation is people diagnosed with bulimia are more likely to be exposed to trauma. Between these two, the most plausible is that people diagnosed with bulimia are more focused on their body and experience anxiety and depression more often. Characteristics of bulimia include an increased focus on the way that a person experiences their body as well as increased comorbidity with anxiety, and mood disorders (Wade, 2019). While someone diagnosed with bulimia might experience trauma, genetic factors are a more significant precursor to the symptomology of bulimia.

The fourth research question explored usage rates of words related to each of Joiner's core IPT dimensions (Joiner, 2009). One credible interpretation of the results is thwarted belongingness and acquired capacity for self-harm or suicide are more frequently found in the experiences of someone diagnosed with bulimia compared to other risk factors. Another solid interpretation is that thwarted belongingness and acquired capacity for self-harm and suicide are more significant compared to other identified risk factors for self-harm or suicide. With regards to these interpretations, the subsequent one is most probable because increased feelings of thwarted belongingness and the physical behavior patterns and symptoms related to bulimia resulting in an increased acquired capacity for suicide, result in a high risk of self-harm or suicide (Joiner et al., 2009).

One limitation of the study is the limited number of memoirs meeting the criteria of the corpus. The limited number of memoirs could be related to misdiagnosis or under diagnosis of bulimia, comorbid diagnosis being emphasized in memoirs rather than bulimia diagnosis, and social stigma that surrounds bulimia. A larger corpus would allow researchers to draw conclusions about the population with more confidence.

A second limitation of the study is the impact the diagnostic criteria for eating disorders has on the corpus. The memoirs included in the corpus predate the revisions made to the Diagnostic Statistical Manual diagnostic criteria for eating disorders, including bulimia (American Psychiatric Association, 2013). Some of the author's experiences included in the corpus might no longer fit the criteria for bulimia. Additionally, Miller and Golden (2010) found that a significant percentage of people diagnosed with an eating disorder will experience crossover symptoms with another eating disorder diagnosis in their lifetime. These dynamics create an obstacle to defining a corpus reflective of the experiences of authors.

This study has implications in the areas of identification of risk of suicide in people diagnosed with bulimia, treatment, the current application of the definition of bulimia, and the need for future research. This study emphasizes the presence of 
thwarted belongingness and acquired capacity for suicide within the experience of people diagnosed with bulimia. Therefore, the need for treatment addressing thwarted belongingness and acquired capacity is highlighted by the findings of this study.

Findings from the study accentuated the impact that the revision of the diagnostic criteria for bulimia plays on diagnosis, treatment, and prevention of bulimia. As the way that the diagnosis is applied by professionals and patients changes, these changes will be reflected in the way that people self-identify as having bulimia, treatment modalities, and understanding risk factors of someone diagnosed with bulimia. These changes indicate a need for future research with a larger corpus, providing a greater depth of understanding of the experiences of someone diagnosed with bulimia.

This study stressed the risk that someone diagnosed with bulimia is at for selfharm or suicide, while specifying thwarted belongingness and acquired capacity for suicide as significant risk factors. This study also provides support for the need to add to the current body of research literature applying the current eating disorder paradigm.

\section{References}

American Psychiatric Association. (2013). Diagnostic and statistical manual of mental disorders (5th ed.). Arlington, VA: Author.

Arcelus, J., Mitchell, A. J., Wales, J., \& Nielsen, S. (2011). Mortality rates in patients with anorexia nervosa and other eating disorders: A meta-analysis of 36 studies. Archives of General Psychiatry, 68, 724-731. https://doi.org/10.1001/archgenpsychiatry.2011.74

Armstrong, S., A. (2009). Not all black girls know how to eat: A story of bulimia. Chicago, IL: Lawrence Hill Books.

Brausch, A. M., \& Perkins, N. M. (2018). Nonsuicidal self-injury and disordered eating: Differences in acquired capability and suicide attempt severity. Psychiatry Research, 266, 72-78. https://doi.org/10.1016/j.psychres.2018.05.021

Centers for Disease Control and Prevention. (2018). Web-based Injury Statistics Query and Reporting System. Atlanta, GA: National Center for Injury Prevention and Control. Retrieved from https://www.cdc.gov/injury/wisqars/index.html

Cohen, J. (1992). A power primer. Psychological Bulletin, 112, 155-159. https://doi.org/10.1037/0033-2909.112.1.155

Coppersmith, G., Dredze, M., Harman, C., \& Hollingshead, K. (2015). From ADHD to SAD: Analyzing the language of mental health on Twitter through self-reported diagnoses. In Proceedings of the 2nd Workshop on Computational Linguistics and Clinical Psychology: From Linguistic Signal to Clinical Reality (pp. 1-10). Stroudsburg, PA: Association for Computational Linguistics. https://doi.org/10.3115/v1/W15-1201

Coppersmith, G., Ngo, K., Leary, R., \& Wood, A. (2016). Exploratory analysis of social media prior to a suicide attempt. In Proceedings of the Third Workshop on Computational Linguistics and Clinical Psychology (pp. 106-117). San Diego, CA. https://doi.org/10.18653/v1/W160311

Fabozzi, F. J., Focardi, S. M., Rachev, S. T., \& Arshanapalli, B. G. (2014). The basics of financial econometrics: Tools, concepts, and asset management applications. Hoboken, NJ: John Wiley \& Sons.

Faul, F., Erdfelder, E., Buchner, A., \& Lang, A. (2009). Statistical power analyses using $\mathrm{G} *$ Power 3.1: Tests for correlation and regression analyses. Behavior Research Methods, 41, 1149-1160. https://doi.org/10.3758/BRM.41.4.1149 
Fernández-Cabana, M., Ceballos-Espinoza, F., Alves-Pérez, M. T., Garcia-Garcia, M. J., Mateos, R. (2013). Suicidal Traits in Marilyn Monroe's Fragments. Crisis, 34(2), 124-130. https://doi.org/10.1027/0227-5910/a000183

Gay, R. (2017). Hunger: A memoir of my body. New York, NY: Harper.

Gunsch, M., Brownlow, S., Haynes, S., \& Mabe, Z. (2000). Differential linguistic content of various forms of political advertising. Journal of Broadcasting \& Electronic Media, 44(1), 27-42. https://doi.org/10.1207/s15506878jobem4401 3

Handelman, L. D., \& Lester, D. (2007). The content of suicide notes from attempters and completers. Crisis: The Journal of Crisis Intervention and Suicide Prevention, 28(2), 102-104. https://doi.org/10.1027/0227-5910.28.2.102

Hill, R. M., \& Pettit, J. W. (2014). Perceived burdensomeness and suicide-related behaviors in clinical samples: Current evidence and future directions. Journal of Clinical Psychology, 70(7), 631-643. https://doi.org/ 10.1002/jclp.22071

Holme, N. (2012). Lesbian crushes and bulimia: A diary of how I acquired my eating disorder. Scotts Valley, CA: Create Space Independent Publishing Platform.

Hudson, J. I., Hiripi, E., Pope, H. G., Jr., \& Kessler, R. C. (2007). The prevalence and correlates of eating disorders in the National Comorbidity Survey Replication. Biological psychiatry, 61(3), 348-358. https://doi.org/10.1016/j.biopsych.2006.03.040

Joiner, T. (2009). The interpersonal theory of suicide: Guidance for working with suicidal clients. Washington, DC: American Psychological Association. http://doi.org/10.1037/11869-000

Joiner, T. E., van Orden, K. A., Witte, T. K., Selby, E. A., Ribeiro, J. D., Lewis, R., \& Rudd, M. D. (2009). Main predictions of the interpersonal-psychological theory of suicidal behavior: Empirical tests in two samples of young adults. Journal of Abnormal Psychology, 118, 634-646. https://doi.org/10.1037/a0016500

Kindle Converter (Version 3.19.918.386) [Computer software]. (2019). Retrieved from https://www.ebook-converter.com

Lewitzka, U., Denzin, S., Sauer, C., Bauer, M., \& Jabs, B. E. (2016). Personality differences in early versus late suicide attempters. BMC Psychiatry, 16(282). https://doi.org/10.1186/s12888-016-0991-6

Marnell, C. (2017). How to murder your life: A memoir. New York, NY: Simon and Schuster.

Mehler, P. S., \& Rylander, M. (2015). Bulimia nervosa - medical complications. Journal of Eating Disorders, 3(12). https://doi.org/10.1186/s40337-015-0044-4

Miller, C. A., Golden, N. H. (2010). An Introduction to Eating Disorders: Clinical Presentation, Epidemiology, and Prognosis, Nutrition and Clinical Practice, 25(2), 110-115. https://doi.org/10.1177/0884533609357566

Pennebaker, J. (2010). Expressive writing in a clinical setting. Journal of Child and Adolescent Psychopharmacology, 18, 573-588. https://doi.org/10.1007/978-0387-29986-0_14

Pennebaker, J. (2017). Mind mapping: Using everyday language to explore social \& psychological processes. Procedia Computer Science, 118, 100-107. https://doi.org/10.1016/j.procs.2017.11.150

Pennebaker, J. W., Booth, R. J., Boyd, R. L., \& Francis, M. E. (2015). Linguistic inquiry and word count: LIWC2015. Austin, TX: Pennebaker Conglomerates.

Pennebaker, J. W., Boyd, R. L., Jordan, K., \& Blackburn, K. (2015). The development and psychometric properties of LIWC2015. Austin, TX: Pennebaker Conglomerates. Retrieved from http://hdl.handle.net/2152/31333 
Sagar, A. (2005). Long term health risks due to impaired nutrition in women with a past history of bulimia nervosa. Nutrition Noteworthy, 7(1). Retrieved from https://escholarship.org/uc/item/6vt2k42t

Silva, C., Ribeiro, J. D., \& Joiner, T. E., Jr. (2015). Mental disorders and thwarted belongingness, perceived burdensomeness, and acquired capability for suicide. Psychiatry Research, 226(1), 316-327. https://doi.org/10.1016/j.psychres.2015.01.008

Suokas, J. T., Suvisaari, J. M., Grainger, M., Raevuori, A., Gissler, M. \& Haukka, J. (2014). Suicide attempts and mortality in eating disorders: A follow-up study of eating disorder patients. General Hospital Psychiatry, 36, 355-357. https://doi.org/10.1016/j.genhosppsych.2014.01.002

Tausczik, Y. R., \& Pennebaker, J. W. (2010). The psychological meaning of words: LIWC and computerized text analysis methods. Journal of Language and Social Psychology, 29(1), 24-54. https://doi.org/10.1177\%2F0261927X09351676

Waters, L. (2011). My secret life: A memoir of bulimia. Dublin, Ireland: Maverick House.

Weisser, M. (2016). Practical corpus linguistics: An introduction to corpus-based language analysis. Malden, MA: Wiley-Blackwell.

Wertheimer, A. (2014). A special scar: The experiences of people bereaved by suicide. New York, NY: Routledge. 
Table 1

Custom Dictionaries

\begin{tabular}{lll}
\hline \multicolumn{1}{c}{ Thwarted Belongingness } & \multicolumn{1}{c}{ Perceived Burdensomeness } & \multicolumn{1}{c}{ Acquired Capacity } \\
\hline single* & hopel* & attempt* \\
reject & ashamed & ideation \\
lone* & worth1* & pain \\
abandon* & doubt & cut* \\
dump* & rely & burn* \\
neglect* & guilty & hunger \\
forgot* & helpl* & scratch* \\
empty & usel* & blood \\
left & waste & vomit* \\
distant & difficult & hurt* \\
homesick & & faint* \\
overlook* & & bone \\
\hline
\end{tabular}

Note. Asterisk notes wildcard was used that counted all endings of a stem. 
Table 2

LIWC Selected Subdictionary Usage Rates

\begin{tabular}{lccc}
\hline \multicolumn{1}{c}{ Variable } & Category & Count* & \% All Words* \\
\hline $1^{\text {st }}$ person singular & Linguistic & 6889 & $8.78 \%$ \\
Biological & Psych./Physical & 3039 & $3.88 \%$ \\
$1^{\text {st }}$ person plural & Linguistic & 1841 & $2.35 \%$ \\
Negative emotion & Psych./Physical & 1826 & $2.33 \%$ \\
Positive emotion & Psych./Physical & 2059 & $2.26 \%$ \\
$3^{\text {rd }}$ person singular & Linguistic & 575 & $0.73 \%$ \\
$3^{\text {st }}$ person plural & Linguistic & 535 & $0.68 \%$ \\
Anger & Psych./Physical & 444 & $0.57 \%$ \\
Risk & Psych./Physical & 447 & $0.57 \%$ \\
Sadness & Psych./Physical & 439 & $0.56 \%$ \\
Anxiety & Psych./Physical & 372 & $0.47 \%$ \\
\hline
\end{tabular}

*Results reported are averages for the five memoirs. 
Table 3

Results Comparisons to Known Norms

\begin{tabular}{lccccccc}
\hline \multicolumn{1}{c}{ Variable } & $\begin{array}{c}\text { Bulimia } \\
\text { Count }\end{array}$ & $\begin{array}{c}\text { Expected } \\
\text { Count }\end{array}$ & $\begin{array}{c}\text { Norm } \\
\text { Count }\end{array}$ & $\begin{array}{c}\text { Expected } \\
\text { Count }\end{array}$ & LL & BIC & $\begin{array}{c}\text { BIC } \\
\text { Description }\end{array}$ \\
\hline $1^{\text {st }}$ person singular & 6889 & 4688.64 & 1728 & 3928.36 & 2463.28 & 2451.4 & Very Strong \\
$1^{\text {st }}$ person plural & 1841 & 2718.4 & 3154 & 2277.6 & 618.56 & 606.68 & Very Strong \\
Biological & 3039 & 2429.47 & 1426 & 2035.53 & $345.6^{*}$ & 333.73 & Very Strong \\
$3^{\text {rd }}$ person plural & 535 & 620.29 & 605 & 519.71 & 25.59 & 13.72 & Very Strong \\
Negative emotion & 1826 & 1737.36 & 1367 & 1455.64 & 9.96 & -1.92 & Strong \\
$3^{\text {rd }}$ person singular & 575 & 531.06 & 401 & 444.94 & 8.03 & -3.85 & Trivial \\
Positive emotion & 2059 & 2074.71 & 1755 & 1738.29 & 2.28 & -9.6 & Trivial \\
Anger & 444 & 423.87 & 335 & 355.13 & 2.11 & -9.77 & Trivial \\
Risk & 447 & 432.57 & 348 & 362.43 & 1.06 & -10.82 & Trivial \\
Anxiety & 372 & 359.66 & 289 & 301.34 & 0.93 & -10.95 & Trivial \\
Sadness & 439 & 435.29 & 361 & 364.71 & 0.07 & -11.81 & Trivial \\
\hline
\end{tabular}

Note. Family-wise error rate $=.05$, adjusted error rate $=.0045, D f=1$, critical value $=8.05$ 
Table 4

LIWC Custom Dictionary Usage Rates

\begin{tabular}{llll}
\hline Variable & Category & Count & \% All Words \\
\hline Thwarted & Custom & 119 & $0.15 \%$ \\
Acquired capacity & Custom & 106 & $0.14 \%$ \\
Burdensomeness & Custom & 74 & $0.09 \%$ \\
\hline
\end{tabular}

Note. Results reported are averages for the five memoirs. 